PSS PROCEEDINGS

\title{
Epoch of Reionization
}

\section{Benedetta Ciardi ${ }^{* \dagger}$}

Max Planck Institute

E-mail: ciardi@mpa-garching.mpg.de

First MCCT-SKADS Training School

September 23-29, 2007

Medicina, Bologna Italy

\section{* Speaker.}

${ }^{\dagger}$ A footnote may follow. 


\section{Introduction}

In these lessons I will discuss about present observational constraints on the reionization process, its theoretical modeling and the possibility of observing it through the $21 \mathrm{~cm}$ line.

After the Big Bang, the universe is filled with hot plasma, but following expansion, the gas cools and eventually becomes neutral about $350.000 \mathrm{yr}$ after the Big Bang. The first structures begin to form at redshift 20-40 (depending on the cosmological model) following the collapse of perturbations in the density field. This marks the beginning of the reionization process, which is completed by $z \sim 6$.

Why do we say that the universe is ionized? This statement follows observations of high redshift QSOs spectra, that show peculiar absorption features at frequencies larger than the Ly $\alpha$ emission line, which are believed to originate from neutral hydrogen along the line of sight of the QSO. From the standard equation of radiative transfer, the intensity $I_{a b s}$ absorbed by a patch of neutral hydrogen with optical depth $\tau_{H I}$ is given by:

$$
I_{a b s}=I_{i n}\left(1-\mathrm{e}^{-\tau_{H I}}\right),
$$

where $I_{\text {in }}$ is the incident radiation. Thus, for $\tau_{H I}=1$ about $63 \%$ of the incident radiation gets absorbed. The optical depth of $\mathrm{HI}$ at mean density at redshift $z$ is:

$$
\tau_{H I}(z) \sim 6.5 \times 10^{5} \frac{n_{H I}}{n_{H}}\left(\frac{1+z}{10}\right)^{1.5},
$$

where $n_{H I}$ and $n_{H}$ are the number density of $\mathrm{HI}$ and $\mathrm{H}$ respectively. The above equation shows that a tiny fraction of HI would completely absorb the flux emitted by the QSO. Thus, the fact that we observe Ly $\alpha$ emission indicates that the intergalactic medium (IGM) is in a highly ionization state.

In the following I will discuss more in detail the present observational constraints on the reionization process, the ingredients needed for its theoretical modeling and the possibility of detecting it via observations of $21 \mathrm{~cm}$ line from neutral hydrogen.

\section{Observational constraints}

In this Section I will briefly summarize the main observational constraints presently available on the reionization process.

Important constraints come from observations of high- $z$ QSOs' spectra. A collection of such spectra (from $z=5.71$ to $z=6.42$ ) is shown in Figure 1. Their analysis shows that (i) the IGM is highly ionized at $z<6$, and (ii) the amount of $\mathrm{HI}$ increases with increasing $z$. A quantitative estimate of the Ly $\alpha$ optical depth (and the associated abundance of neutral hydrogen) is used to retrieve information on the latest stages of the reionization process (e.g. Fan et al. 2006). Additional information comes from observations of the cosmic microwave background radiation (CMB). The electrons produced during reionization interact with the photons of the CMB and induce anisotropies in its power spectrum. The intensity of the anisotropies is typically described in terms of the Thomson scattering optical depth:

$$
\tau_{t h}(z) \propto \int n_{e}\left(z^{\prime}\right) d z^{\prime},
$$




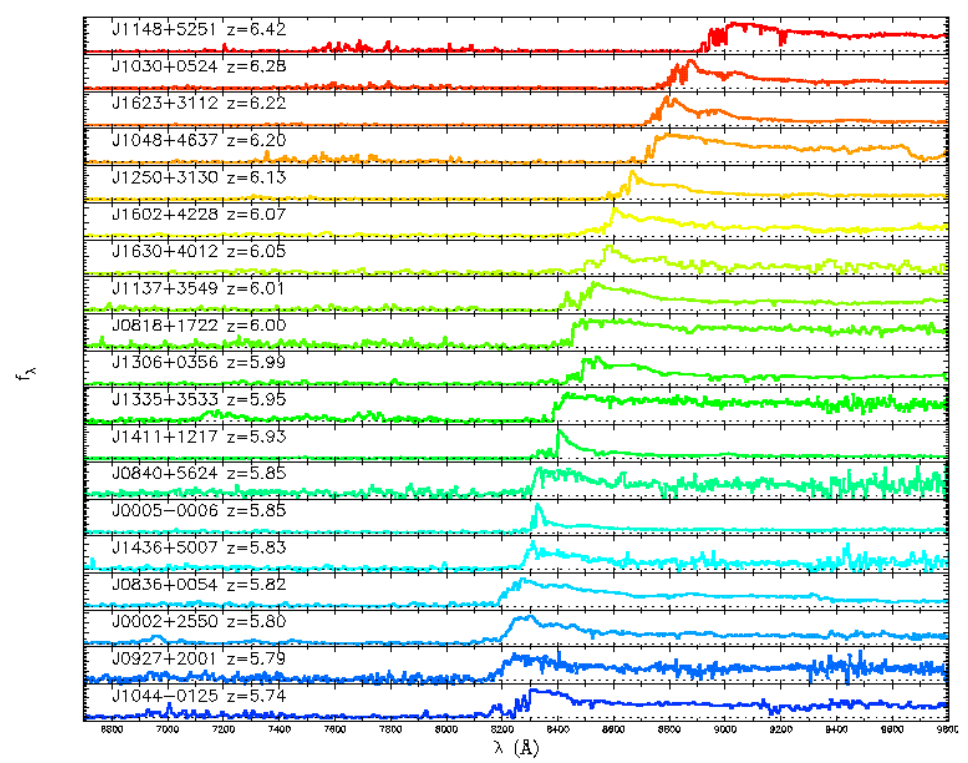

Figure 1: Absorption spectra of QSOs with $5.7<z<6.42$ (Fan).

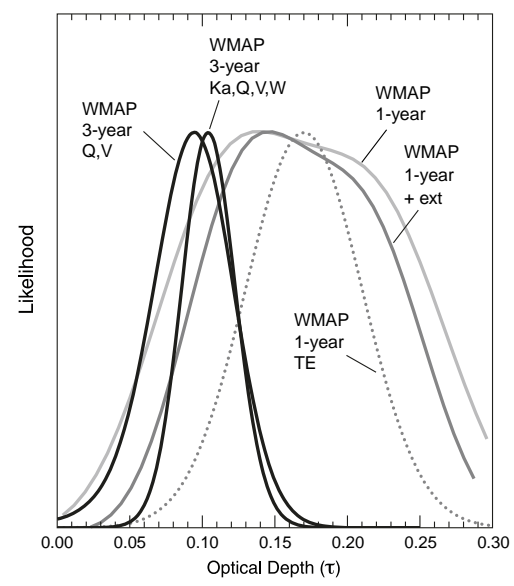

Figure 2: Likelywood curves for the Thomson scattering optical depth as measured from the WMAP satellite (for details see Page et al. 2007).

where $n_{e}$ is the number density of electrons. In Figure 2 likelywood curves for $\tau_{t h}$ are shown as measured from the WMAP satellite (Page et al. 2007). These observations provide an estimate of the global amount of electrons produced during reionization, but no information is given on the reionization redshift $z_{i o n}$, as different reionization histories can produce the same optical depth but different $z_{i o n}$.

To summarize, at present we have information on the latest stages of reionization and on the global amount of electrons produced during this process, but e.g. the nature of the sources responsible for the IGM reionization or the precise reionization history are not known. 


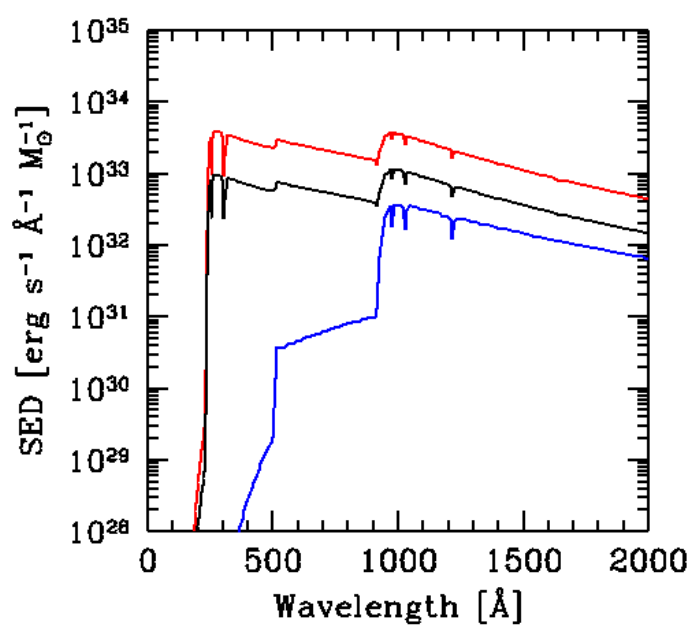

Figure 3: Spectral energy distribution for different stellar populations. The bottom line represent a stellar population with metallicity $Z=10^{-2} Z_{\odot}$ and a Salper IMF in the range $0.1-120 \mathrm{M}_{\odot}$. The middle and upper lines are for metal-free stars with a Salpeter and a Larson IMF respectively, in the same stellar mass range.

\section{Theoretical models}

The ingredients needed to model the reionization process are (i) a model of galaxy formation, (ii) the properties of the reionization sources and (iii) a prescription to follow the propagation of the ionizing photons.

Despite many applications of hydrodynamical simulations to the structure formation process, much of our current understanding comes from semi-analytical models, based on simplified physical assumptions. These models have the advantage of allowing a larger dynamic range and of being fast enough to explore a vast range of parameters. Their main disadvantage is that no information on the spatial distribution of structures is provided. For this reason a method has been developed that combines N-body simulations of the dark matter component, with semi-analytical models predicting galactic properties "a posteriori". The limitation of the above methods is related to the resolution of the N-body simulations, which turns out to be critical in studies of the reionization process. At the present state, while the evolution of dark matter structures is well understood, the treatment of the physical processes that govern the formation and evolution of the luminous objects (e.g. heating/cooling of the gas, star formation, feedback) is more uncertain. In particular, no study treats the entire range of the feedback effects that can influence the galaxy formation process. The formation and evolution of early structures is heavily affected by feedback effects. In fact, once the first sources are formed, their mass deposition, energy injection and emitted radiation can deeply affect the subsequent galaxy and star formation process, and the evolution of the IGM. Generally speaking, feedback effects can either reduce (negative feedback) or increase (positive feedback) the efficiency of the star formation (SF) process. Although a rigorous classification is not feasible because such effects are produced by the same sources and it is hard to separate their individual impact, feedback can be divided into three broad classes (for a review on the topic see Ciardi \& Ferrara 2005; Ciardi 2007): mechanical, chemical and radiative. A first attempt to treat 

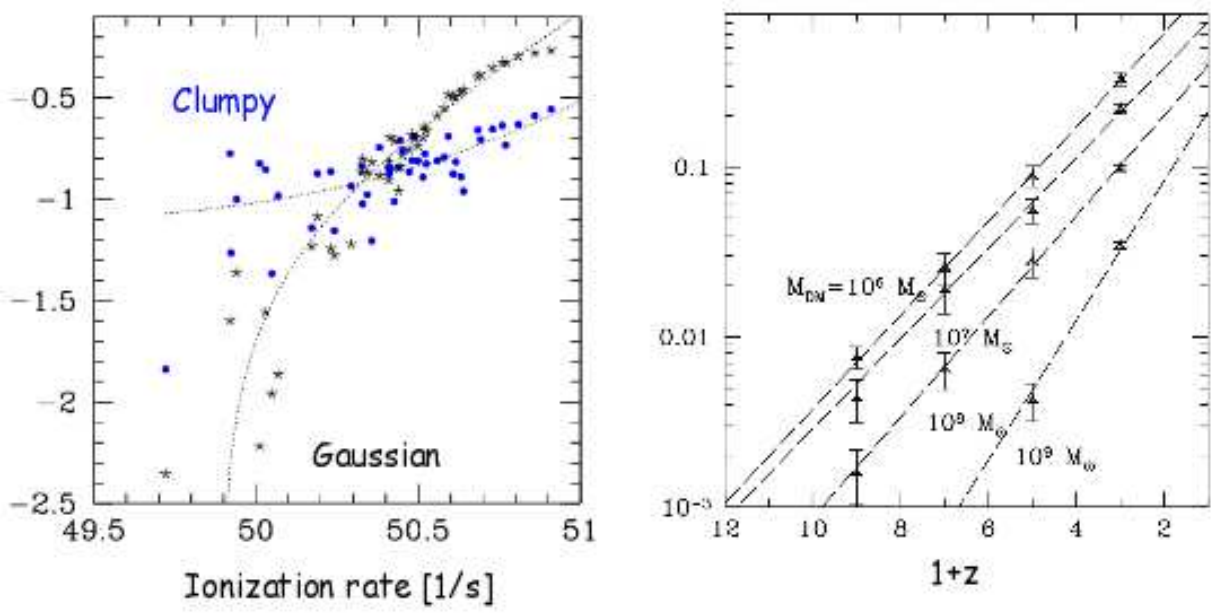

Figure 4: Left panel: Escape fraction as a function of the total ionization rate. Stars (circles) indicate a Gaussian (clumpy) distribution of the gas inside the host galaxy. For details see Ciardi, Bianchi \& Ferrara (2002). Right panel: Escape fraction as a function of redshift. Different curves refer to different host halo mass as indicated. For details see Ricotti \& Shull (2000).

self-consistently a number of feedback effects ranging from the mechanical energy injection to the $\mathrm{H}_{2}$ photodissociating radiation produced by massive stars has been done by Ciardi et al. (2000), followed by the more recent Ricotti, Gnedin \& Shull (2002) and Yoshida et al. (2003), but the complexity of the network of processes makes their implementation not trivial.

Regarding the sources of ionizing radiation, they can be of stellar type, quasars or more exotic sources as e.g. dark matter decay or annihilation. The uncertainties associated with their properties are large. Also if we concentrate our attention on stellar type sources, we do not have much information on their initial mass function (IMF) and spectrum. An example of different spectra is shown in Figure 3. The bottom line represents a stellar population with metallicity $Z=10^{-2} Z_{\odot}$ and a Salper IMF in the range $0.1-120 \mathrm{M}_{\odot}$. The middle and upper lines are for metal-free stars with a Salpeter and a Larson IMF respectively, in the same stellar mass range ${ }^{1}$. From the Figure it is clear that the ionizing photon output can be very different for different stellar populations.

Another critical quantity is the escape fraction, $f_{e s c}$, i.e. the fraction of ionizing photons which are able to escape from the galaxy where they are produced. This quantity is extremely uncertain and depends on a variety of quantities, e.g. the gas distribution, the mass and redshift of the host galaxy (Fig. 4; Dove, Shull \& Ferrara 2000; Ricotti \& Shull 2000; Wood \& Loeb 2000; Ciardi, Bianchi \& Ferrara 2002; Fujita et al. 2003; Yoshida et al. 2007).

The situation can be much different in the case of very massive, primordial stars hosted by small mass halos, for which $f_{\text {esc }}$ is larger than $70 \%$ (Fig. 5).

Also observations provide a very large range of $f_{\text {esc }}$, with values as small as a few percent and as high as $\sim 50 \%$ (e.g. Leitherer et al. 1995; Hurwitz et al. 1997; Bland-Hawthorn \& Maloney 1999; Steidel, Pettini \& Adelberger 2001).

\footnotetext{
${ }^{1}$ For a Salpeter IMF the number of stars per logarithmic unit of stellar mass is given by $d N / d \log M_{\star} \propto M_{\star}^{-1.35}$, while for a Larson IMF it is $d N / d \log M_{\star} \propto\left(1+M_{\star} / M_{c r i t}\right)^{-1.35}$, where $M_{c r i t}$ is a parameter.
} 


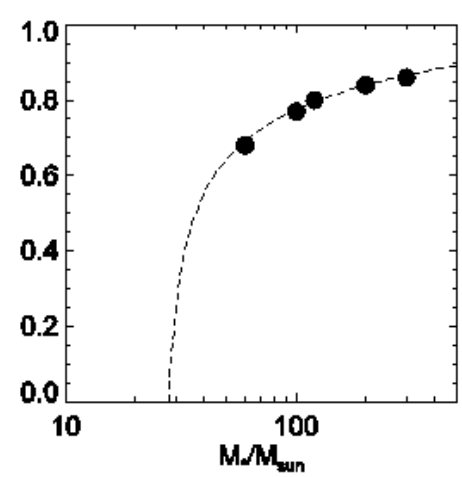

Figure 5: Escape fraction as a function of stellar mass. For details see Yoshida et al. (2007).

Finally, the propagation of ionizing radiation is generally followed using radiative transfer codes. The radiative transfer equation in a cosmological context is:

$$
\frac{1}{c} \frac{\delta I_{v}}{\delta t}+\frac{\mathbf{n} \times \nabla I_{v}}{a_{e m}}-\frac{H(t)}{c}\left(v \frac{\delta I_{v}}{\delta v}-3 I_{v}\right)=\eta_{v}-\chi_{v} I_{v}
$$

where $I_{v}$ is the flux intensity at frequency $v, a_{e m}=\left(1+z_{e m}\right) /(1+z)$ is the expansion factor, $H(t)$ is the Hubble parameter, $\eta_{v}$ is the emission coefficient and $\chi_{v}$ is the absorption coefficient. The second term on the left hand side of the equation represents the path length change due to the expansion, the third term the redshift of the radiation and the fourth the dilution due to expansion. The above is a seven dimension equation with no symmetry. In typical situations $a_{e m} \sim 1$ and if the local approximation is adopted the equation reduces to:

$$
\frac{1}{c} \frac{\delta I_{v}}{\delta t}+\mathbf{n} \times \nabla I_{v}=\eta_{v}-\chi_{v} I_{v} .
$$

As only few simple test cases have an analytical solution (e.g. the formation and evolution of an ionized region around a source embedded in a medium of constant density; Shapiro \& Giroux 1987), recently the cosmological community has gathered to compare the available codes by solving common test cases (for details see Iliev et al. 2006).

Using the ingredients discussed above, models of reionization histories can be built. A typical example is shown in Figure 6.

In Figure 7 a quantitative representation of different reionization histories is shown in terms of the evolution of the volume averaged ionization fraction (inset) and the Thomson scattering optical depth (main figure). From the Figure is it clear that models can easily match the observed values of the Thomson scattering optical depth. At this stage we can try to improve the modeling of the sources or include better physics, but generally it is possible to find a combination of parameters able to match the observed values. For this reason, different type of observations are needed.

\section{The $21 \mathrm{~cm}$ line diagnostic}

The ground state of neutral hydrogen has hyperfine levels depending on whether the spins of the proton and the electron are parallel or anti-parallel. When a spin-flip transition takes place, 


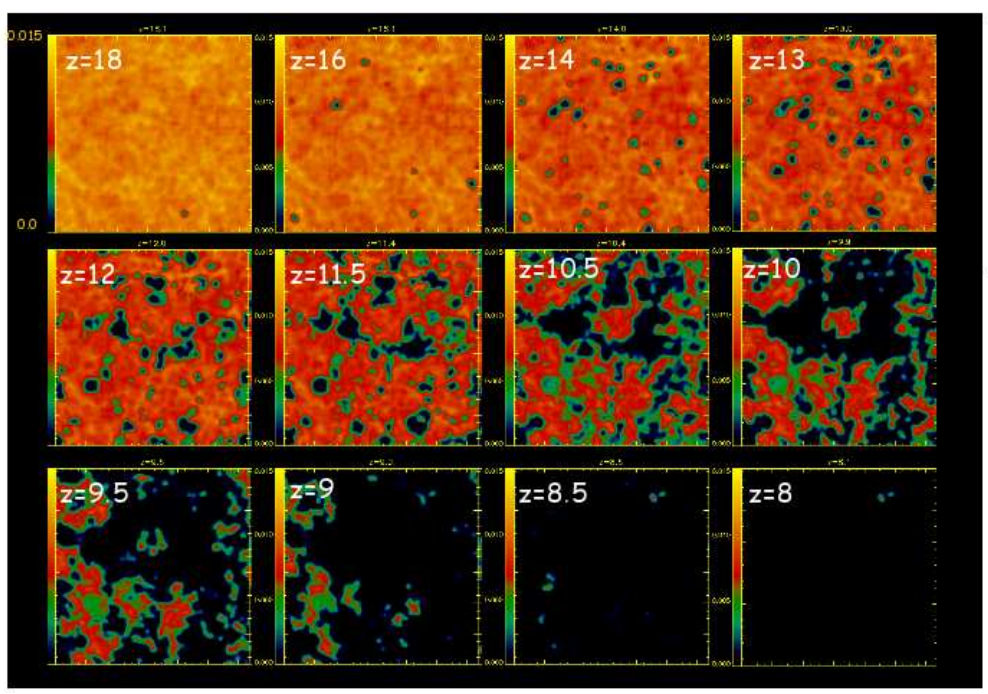

Figure 6: Slices cut through a simulation box, showing the evolution of neutral hydrogen. Different panels indicate different redshifts. In this specific model, the IGM is completely neutral at $z=18$, then, once the first sources are born, ionized regions form around such sources. With time they increase in volume and number, until reionization is completed at $z \sim 8$ (for details see Ciardi, Stoehr \& White 2003).

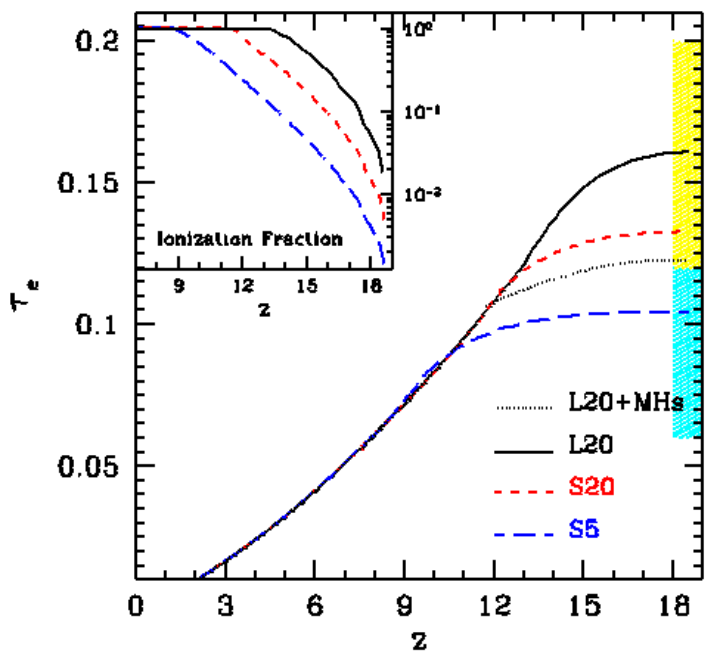

Figure 7: Inset: Evolution of the volume averaged ionization fraction. Main figure: Evolution of the Thomson scattering optical depth. Different lines refer to reionization histories driven by stellar type sources with different properties: Salpeter IMF and $f_{\text {esc }}=5 \%$ (long-dashed lines), Salpeter IMF and $f_{\text {esc }}=20 \%$ (short-dashed lines) and Larson IMF with $f_{\text {esc }}=20 \%$ (solid lines). Dotted lines are the same as solid, with the inclusion of sub-grid physics to take into account the effect of minihalos that can not be resolved by the simulations. The shaded areas indicate the range allowed by the WMAP satellite (Kogut et al. 2003; Spergel et al. 2006). For details see Ciardi, Stoehr \& White (2003), Ciardi, Ferrara \& White (2003), Ciardi et al. (2006). 
photons at $21 \mathrm{~cm}$ are emitted or absorbed. Taking advantage of the shift of the radiation towards the red:

$$
v=v_{e m / a b s}\left(1+z_{e m / a b s}\right)^{-1},
$$

where $v_{e m / a b s}=1415 \mathrm{MHz}$ is the frequency corresponding to $21 \mathrm{~cm}$ and $z_{e m / a b s}$ is the redshift at which the radiation is emitted or absorbed, it is possible to probe the evolution of the abundance of HI. In fact, different observed frequencies correspond to different emission/absorption redshift (e.g. an observed frequency of $130 \mathrm{MHz}$ corresponds to an emission/absorption redshift of about 10). Thus, observations of $21 \mathrm{~cm}$ line from the neutral IGM constitutes the ideal probe of the evolution of the reionization process.

The population of the ground states of HI can be described by the standard Boltzmann equation:

$$
\frac{n_{1}}{n_{0}}=\frac{g_{1}}{g_{0}} \exp \left(-\frac{h v_{21 c m}}{k_{B} T_{s}}\right)=3 \exp \left(-\frac{T_{\star}}{T_{s}}\right),
$$

where $n_{0}$ and $n_{1}$ are the populations in the lower and upper level, $g_{0}$ and $g_{1}$ are the respective statistical weights, $T_{\star}=0.068 \mathrm{~K}, v_{21 \mathrm{~cm}}=1415 \mathrm{MHz}, k_{B}$ is the Boltzmann constant and $T_{s}$ is the spin temperature, which regulates the population of the levels.

Using a combination of the radiative transfer equation and the definition of brightness temperature, and remembering that at radio frequencies the Rayleigh-Jeans regime applies, the differential brightness temperature, $\delta T_{b}$, between the CMB radiation and a patch of HI with optical depth $\tau$ and spin temperature $T_{s}$ is given by (see Furlanetto, Oh \& Briggs 2006 for a review):

$$
\delta T_{b}=T_{b}-T_{C M B} \approx \frac{T_{s}-T_{C M B}}{1+z} \tau .
$$

If $T_{s}=T_{C M B}$ the above equation is equal to zero and in this case we expect to detect no signal. If $T_{C M B}>T_{S}$ the equation is negative and we expect to observe the $21 \mathrm{~cm}$ line in absorption against the CMB, otherwise in emission. Thus, the value of $T_{s}$ is critical for the observability of the line, and in particular it is important for it to be different from $T_{C M B}$.

There are essentially three processes that determine the value of the spin temperature: absorption of CMB photons; collision with $\mathrm{H}$, electrons and protons; Ly $\alpha$ photons' scattering (the Wouthysen-Field effect; Wouthysen 1952; Field 1958). As the relevant timescales for these processes are all much shorter than the expansion time, we can assume an equilibrium configuration to determine the value of $T_{s}$, which is given by a weighted balance between the CMB, collisions and scatterings (Furlanetto, Oh \& Briggs 2006):

$$
T_{s}=\frac{T_{C M B}+\left(y_{\alpha}+y_{c}\right) T_{k}}{1+y_{\alpha}+y_{c}}
$$

where $T_{k}$ is the gas temperature, while $y_{\alpha}$ and $y_{c}$ measure the efficiency of scatterings and collisions respectively.

The general picture that we expect is as follows. At very high redshift the CMB and the gas are coupled due to the scattering between photons and electrons. After recombination though, as the residual fraction of electrons is small, the scattering rate increases and eventually becomes larger than the expansion rate. From this moment on, gas and radiation evolve separately. While $T_{C M B} \propto(1+z)$, the gas temperature $T_{k} \propto(1+z)^{2}$ and drops below $T_{C M B}$. At these high $z$, as the 


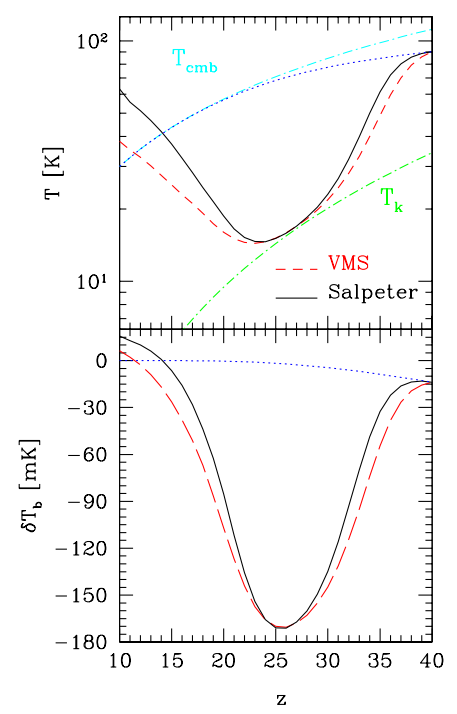

Figure 8: Upper panel: Evolution of the spin temperature for a Salpeter IMF (solid lines) and very massive stars with $M_{\star}=300 \mathrm{M}_{\odot}$ (dashed), and in the absence of a Ly $\alpha$ background (dotted). $T_{\mathrm{CMB}}$ and $T_{k}$ are plotted as upper and lower dotted-dashed lines, respectively. Lower panel: Evolution of the differential brightness temperature. Lines are the same as in the upper panel. See Ciardi \& Salvaterra (2007) for details.

gas density is high, the collision rate is high and, in the absence of Ly $\alpha$ photons, $T_{s}=\left(T_{C M B}+\right.$ $\left.y_{c} T_{k}\right) /\left(1+y_{c}\right) \approx T_{k}$. Thus $\delta T_{b}<0$ and we expect to observe the line in absorption against the CMB (Fig. 8). But at $z<20$ collisions are not efficient anymore, CMB photons are absorbed and a thermal equilibrium with the CMB is quickly reached. So, a mechanism to decouple $T_{s}$ from $T_{C M B}$ is needed. This is the epoch of first structure formation, when the first sources of radiation turn on. In addition to ionizing photons, $\operatorname{Ly} \alpha$ photons are emitted and $\operatorname{Ly} \alpha$ scattering is a very efficient decoupling mechanism. In Figure 9 an estimate of the Ly $\alpha$ background produced by metal-free stars is shown. If we use such estimate to calculate the effect of scattering we see that the $21 \mathrm{~cm}$ line can actually be observed in absorption soon after the first sources have turned on (dotted lines in Fig. 8). During the scattering, photons can transfer energy to the gas and raise its temperature. If $\operatorname{Ly} \alpha$ heating is included in the calculation, the line can be seen in emission at $z<15$ (11) for a Salpeter IMF (very massive stars).

In addition to $\operatorname{Ly} \alpha$ scattering, other sources of heating are available in the high redshift universe, e.g. x-rays from SN remnants or a primordial population of quasars. As the $\mathrm{HI}$ cross section has a strong dependence on frequency $\left(\sigma_{H I} \propto(v / 13.6 \mathrm{eV})^{-3}\right)$, x-ray photons can travel much further away from their production site than UV photons. In addition, they can only partially ionize HI, so that we can expect regions heated by x-ray photons but not completely ionized (e.g. Madau, Meiksin \& Rees 1997; Giroux \& Shull 2001; Glover \& Brand 2001; Chen \& Miralda-Escudé 2004; Gnedin \& Shaver 2004; Zaroubi et al. 2006; Kuhlen \& Madau 2005).

Whatever the source of heating, we can expect to observe the $21 \mathrm{~cm}$ line in emission in the early universe. If $T_{s}>>T_{C M B}$, equation 4.3 becomes:

$$
\delta T_{b} \propto\left(T_{s}-T_{C M B}\right) \frac{n_{H I}}{T_{s}} \propto n_{H I} .
$$




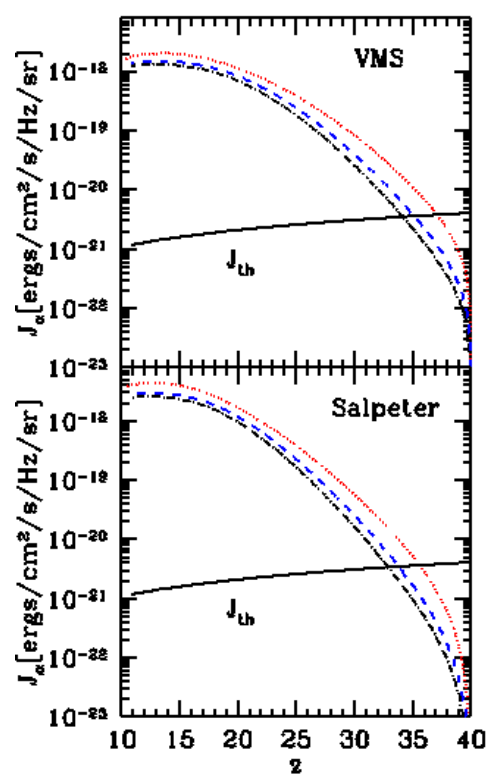

Figure 9: Ly $\alpha$ background produced by metal-free stars with a Salpeter IMF (lower panel) or very massive stars with $M_{\star}=300 \mathrm{M}_{\odot}$ (upper panel). Different lines indicate different models (see Ciardi \& Salvaterra 2007 for details). The solid line marked $J_{t h}$ indicates the threshold value above which the scattering rate is high enough for the decoupling.

The above equation clearly shows the power of radio observations, as observations of $\delta T_{b}$ would give a direct estimate of the abundance of neutral hydrogen at different redshifts. Also from a theoretical point of view, it becomes easy to obtain maps of differential brightness temperature (see Fig. 10) from maps of HI evolution as the one shown in Figure 6.

Prior to structure formation, in the absence of heating sources, we expect to observe the line in absorption. In this case, observations of $\delta T_{b}$ would give information on the underlying density distribution and, thus, cosmological parameters (e.g. Loeb \& Zaldarriaga 2004; Ali, Bharadwaj \& Panday 2005; Barkana \& Loeb 2005; Pillepich, Porciani \& Matarrese 2007; Lewis \& Challinor 2007). An example of the power spectrum of the expected signal at different redshifts is shown in Figure 11.

Once reionization process has started though, maps as those in Figure 10 are expected to be observed. These can be analyzed to retrieve important information. For example, the simulated maps can be convolved with the instrumental characteristics expected for a given radio telescope, to produce mock observed maps as the ones shown in Figure 12. Although in principle feasible, tomography like the one shown in the Figure can be obtained only after several thousands hours of observation, thus, it will not be the short term goal of the planned generation of radio telescopes.

Another interesting application will be observations of $\mathrm{HI}$ around ionized regions produced by QSOs at $z \sim 6$, as the one shown in Figure 13. Such observations would provide additional information on the physical state of $\mathrm{HI}$ during the latest stages of reionization (Zaroubi \& Silk 2005; Wyithe, Loeb \& Barnes 2005; Chen \& Miralda-Escudé 2006; Cen 2006; Rhook \& Haehnelt 2007; Liu et al. 2007). 


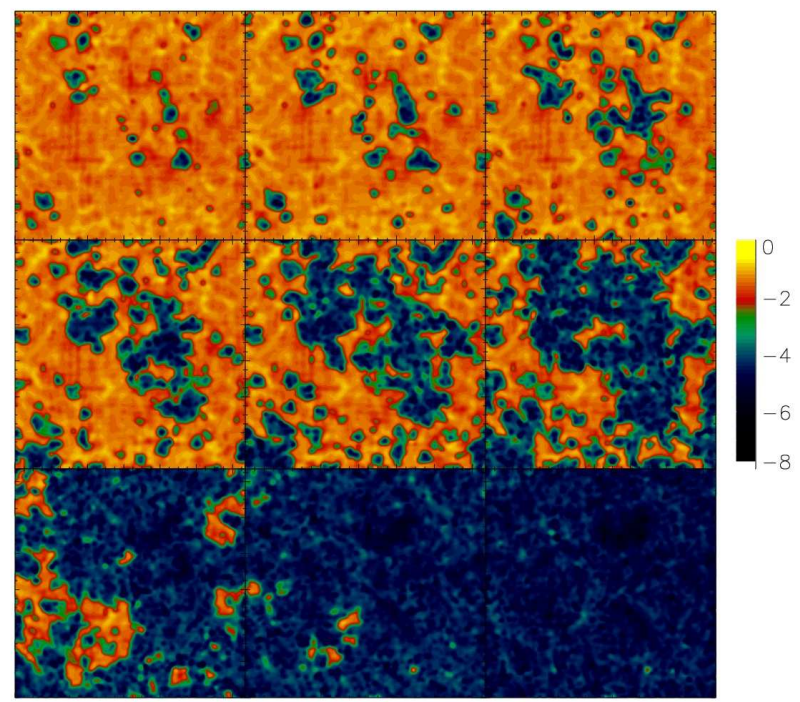

Figure 10: Maps of differential brightness temperature at redshift 13.5, 12.8, 12.0, 11.3, 10.6, 9.9, 9.3, 8.7 and 8.1 (from left to right and top to bottom; Ciardi \& Madau 2003).

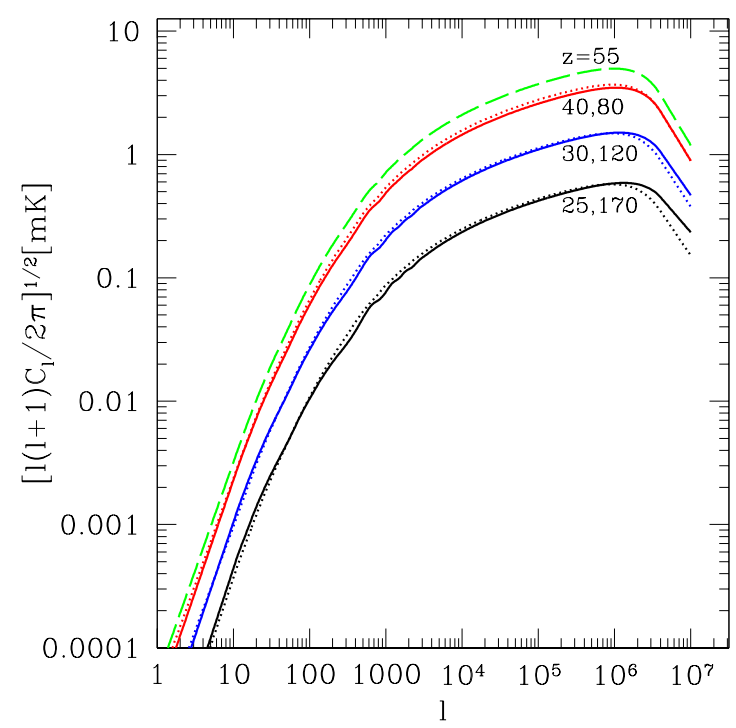

Figure 11: Angular power spectrum of $21 \mathrm{~cm}$ anisotropies on the sky at various redshifts. From top to bottom, $z=55,40,80,30,120,25,170$. For more details see Loeb \& Zaldarriaga (2004).

While HI maps as described above will be difficult to obtain because they require very long integration times, the first observational goals of the planned generation of radio telescopes will be to collect statistical information, e.g. fluctuations of the brightness temperature. In the upper (lower) panel of Figure 14 the r.m.s. of the differential brightness temperature is shown as a function of the observed angle for a "late" ("early") reionization history, for which reionization is complete by $z \sim 13.5$ (8). Different curves refer to different redshift (or observed frequency). The two cases show a similar behavior, with the peak of the emission at $10-30 \mathrm{mK}$. The only difference 


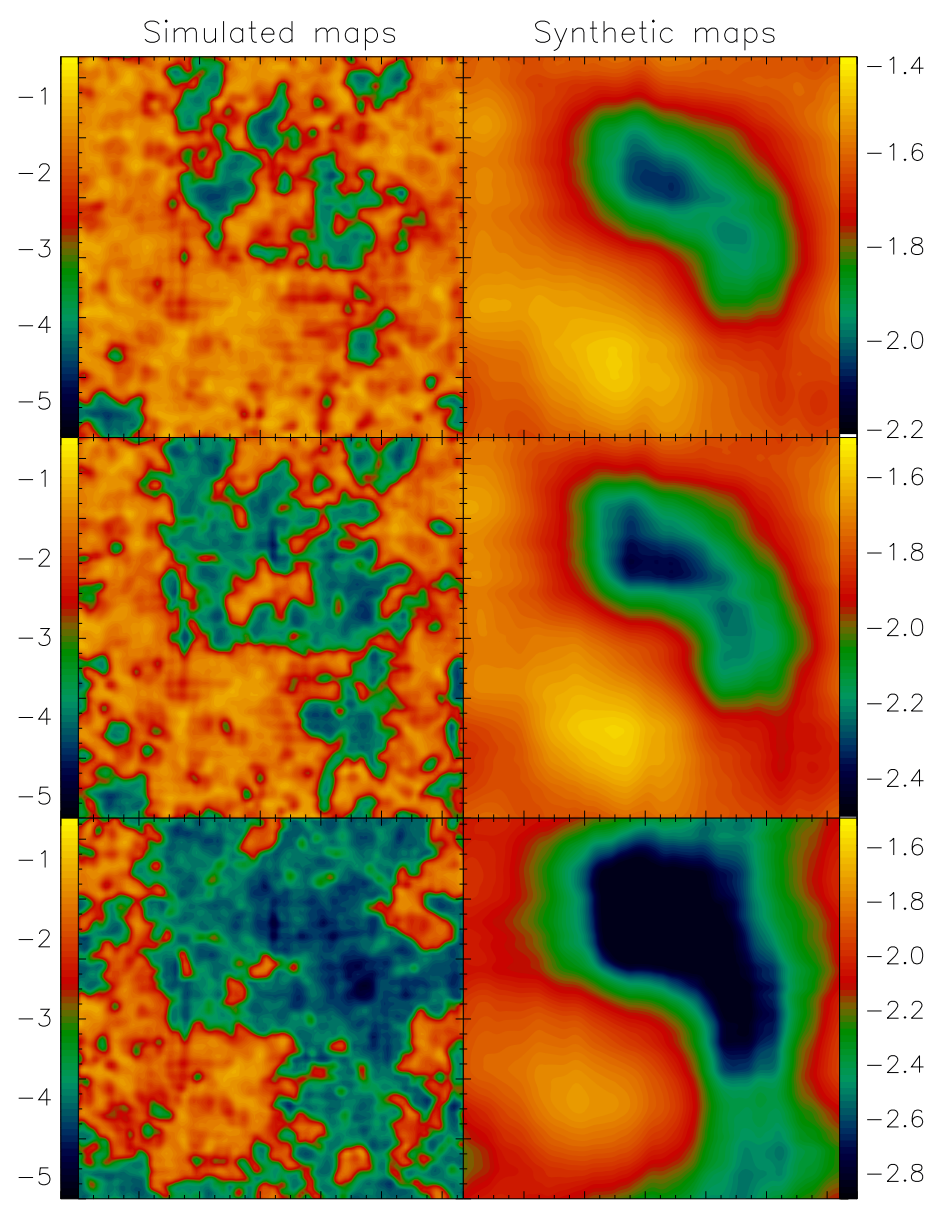

Figure 12: Simulated maps of differential brightness temperature (left panels) compared to maps obtained after convolving the simulated maps with instrumental characteristics (right panels). The top (central, bottom) panels refer to $z=10.6(9.89,9.26)$ or observed frequency $122 \mathrm{MHz}(130 \mathrm{MHz}, 138 \mathrm{MHz})$. For details see Valdes et al. 2006.

is that in the "early" reionization case the curves are shifted towards higher $z$ and thus the peak is at $\sim 90 \mathrm{MHz}$ rather than $\sim 115 \mathrm{MHz}$. In any case, the expected sensitivities of the planned radio telescopes should be enough to detect such signals.

In addition to $21 \mathrm{~cm}$ line in emission, absorption studies can be done along the line of sight of high- $z$ radio sources. Carilli and collaborators (Carilli, Gnedin \& Owen 2002; Carilli et al. 2004) have proposed to observe the $21 \mathrm{~cm}$ forest, in analogy to the Ly $\alpha$ forest, to retrieve additional information on the HI in the IGM (see Fig. 15). As the cross section for $21 \mathrm{~cm}$ absorption is several orders of magnitudes lower than that for Ly $\alpha$ absorption, the $21 \mathrm{~cm}$ forest would probe IGM at a higher density. The uncertainty associated with this kind of observations is on the existence of radio loud sources at very high redshift.

Observations of $21 \mathrm{~cm}$ emission line can give additional information if used in combination with other observations, e.g. of the CMB. As I was mentioning in the introduction, the interaction 

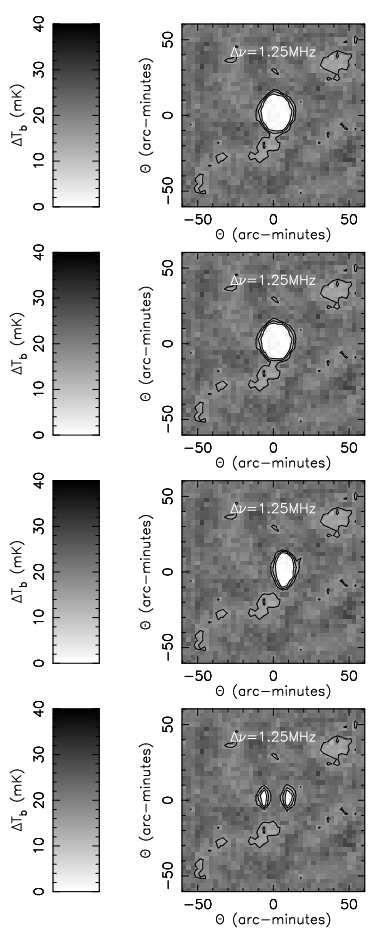
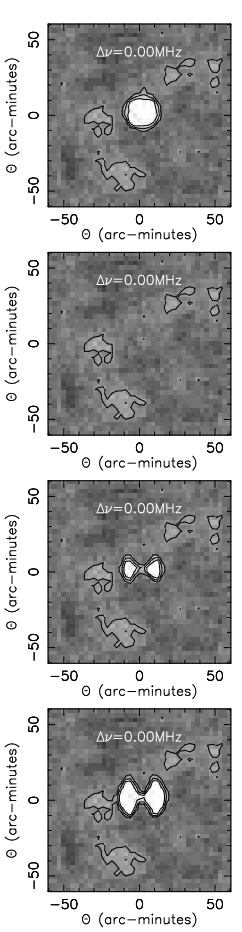
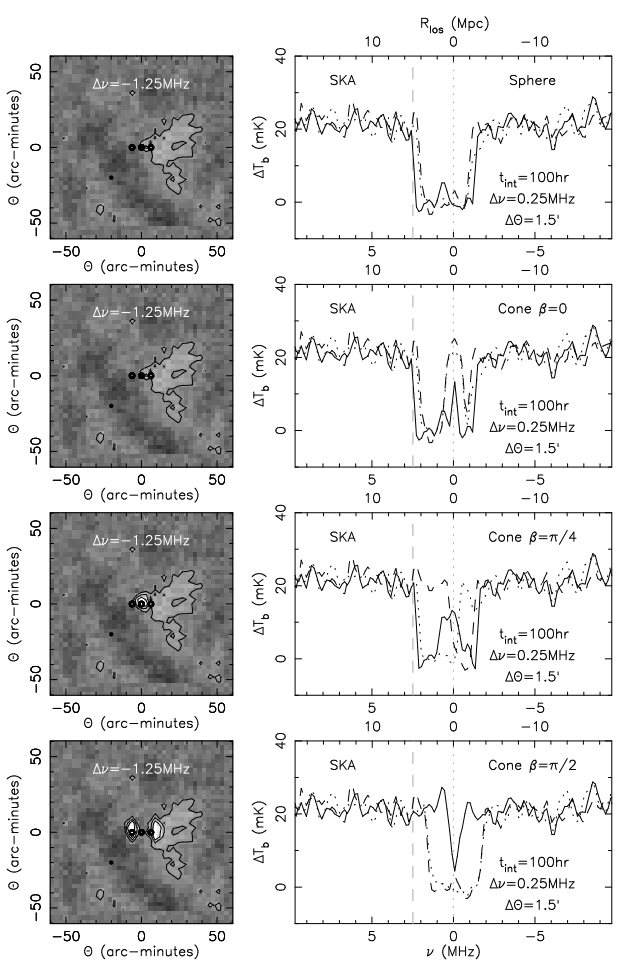

Figure 13: Sample maps of HII regions observed around quasars at $z=6.5$ using the SKA with a $1.5^{\prime}$ Gaussian beam and top-hat smoothed at $0.25 \mathrm{MHz}$ in a $100 \mathrm{hr}$ integration. Four rows are shown. The first row corresponds to observation of an intrinsically spherical HII region. The second, third and fourth rows correspond to observations of an intrinsically bi-conical HII region with an opening angle of $\pi / 3$ oriented along, at $\pi / 4$ to, and perpendicular to the line-of-sight. In each case three slices of the frequency cube are shown. The contours correspond to 5,11 and $17 \mathrm{mK}$. In addition, 3 smoothed spectra are shown in the right-most panel (Wyithe, Loeb \& Barnes 2005).

between the $\mathrm{CMB}$ photons and the electrons produced during reionization induces anisotropies in the CMB power spectrum. Thus, while the CMB anisotropies depend on the amount of electrons produced during reionization, the $21 \mathrm{~cm}$ emission depends on the amount of HI. For this reason, we expect some anti-correlation between the two signals. If maps of CMB anisotropies are correlated with maps of $21 \mathrm{~cm}$ emission, an anti-correlation is found on scales below a characteristic angular scale $\theta$, as shown in Figure 16. Such scale gives an estimate of the typical dimension of the HII regions at the redshift of $21 \mathrm{~cm}$ emission: $\theta$ increases with decreasing redshift (because the HII regions grow with time) and, at the same redshift, it is higher with an "early" reionization history for which the HII regions are typically larger. If we could use a combination of these different observations we would be able to maximize the information in terms of e.g. instrumental noise, systematic errors, foregrounds.

In fact, all the studies mentioned above will be feasible only if the observations will be cleaned from contamination from Galactic and extra-galactic foregrounds. The main contribution comes from synchrotron emission within the Milky Way. As a rule of thumb, the brightness temperature of the sky is $\sim 180(v / 180 \mathrm{MHz})^{-2.6} \mathrm{~K}$ (Furlanetto, Oh \& Briggs 2006), so it is worse at low frequencies. In addition to galactic foregrounds, extra-galactic contamination poses a serious challenge 


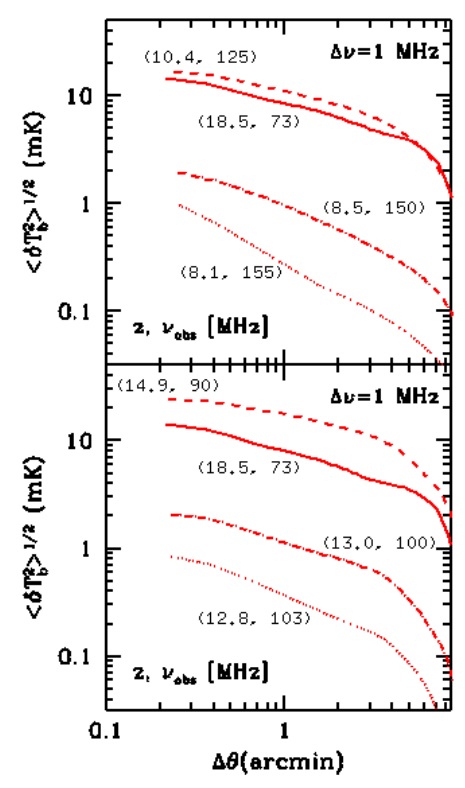

Figure 14: Upper (lower) panel: evolution of the r.m.s. of the differential brightness temperature as a function of the observed angle for a "late" ("early") reionization history, for which reionization is complete by $z \sim 13.5$ (8). Different curves refer to different redshift (or observed frequency; Ciardi \& Madau 2003).
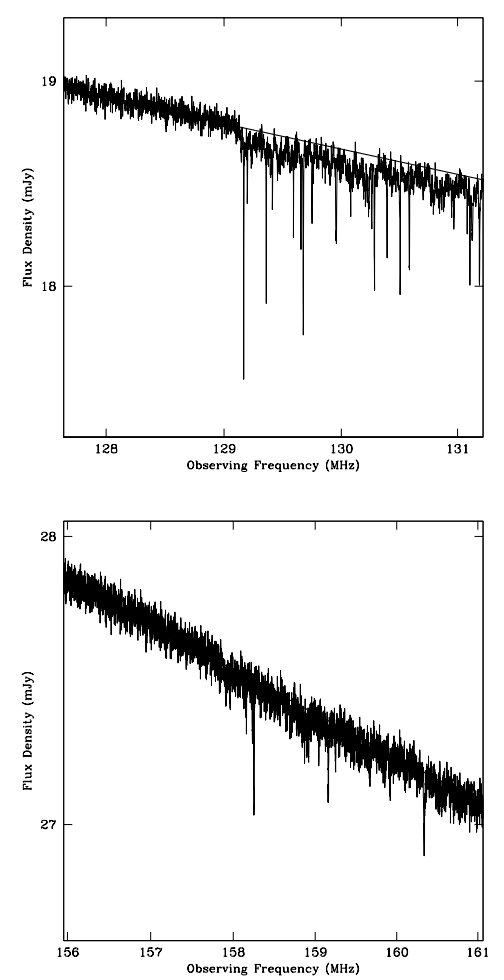

Figure 15: Upper (lower) panel: $21 \mathrm{~cm}$ forest along the line of sight of a radio source with flux at $120 \mathrm{MHz}$ of $20 \mathrm{mJy}$ (35 mJy) located at $z=10(z=8)$. See Carilli, Gnedin \& Owen (2002) for more details. 


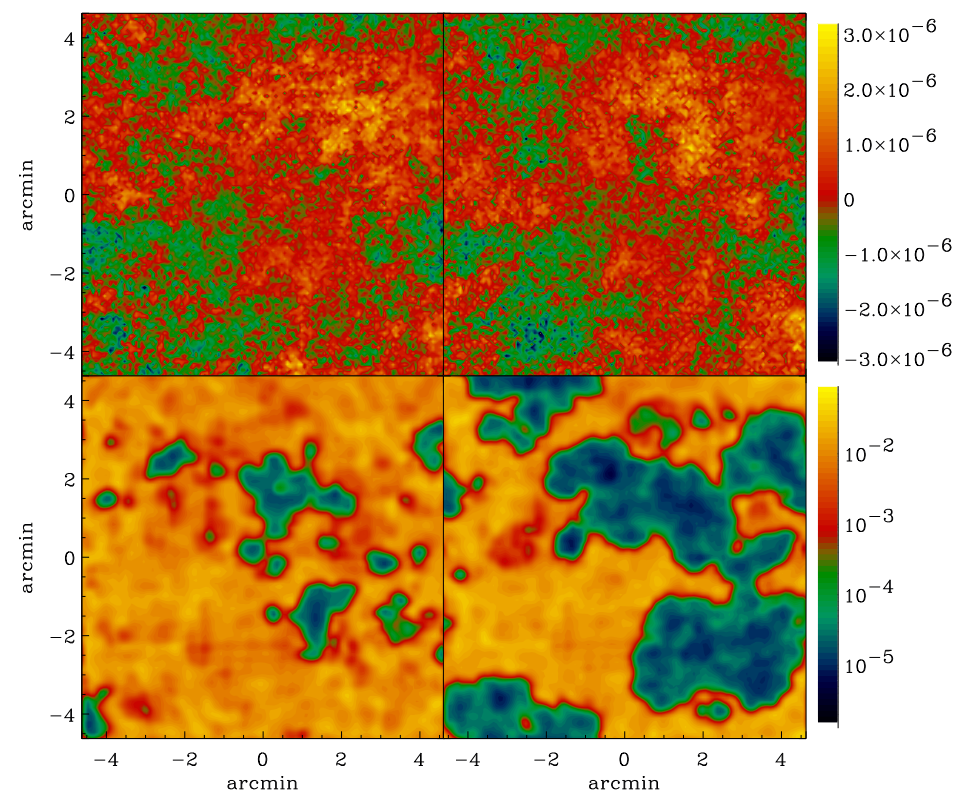

Figure 16: Redshift evolution of the characteristic angular scale for anti-correlation between CMB temperature anisotropies and differential brightness temperature anisotropies. The upper (lower) curves are for an "early" ("late") reionization history with reionization redshift of about 13.5 (8). For details see Salvaterra et al. 2005.

to observations of HI hyperfine emission (e.g. Shaver et al. 1999; Di Matteo et al. 2002, 2004; Oh \& Mack 2003; Cooray \& Furlanetto 2004; Gnedin \& Shaver 2004; Zaldarriaga et al. 2004; Santos et al. 2005; Wang et al. 2006). Examples of such contaminants are free-free emission form interstellar and intergalactic HII regions, low- $z$ radio galaxies, synchrotron emission from clusters (Fig. 17). If bright sources are present they can be removed. In this case the $21 \mathrm{~cm}$ emission line is free from extra-galactic foreground contamination at scales above 1 arcmin (Di Matteo, Ciardi \& Miniati 2004), but additional cleaning is needed (e.g. Santos et al. 2005).

\section{Conclusions}

To summarize, from the present observations of the reionization process we know $(i)$ that the IMG at $z<6$ is in a highly ionization state; (ii) that the amount of HI in the IGM increases with increasing redshift; (iii) an estimate of the global amount of electrons produced during reionization, but no information is available on the nature of the sources of ionizing photons and the actual reionization history.

To retrieve additional information, different observations are needed. Measurements of the $21 \mathrm{~cm}$ line of $\mathrm{HI}$ in absorption or emission will allow to e.g. map the reionization history, have better constraints on the reionization sources, constrain the matter power spectrum and cosmological parameters. 


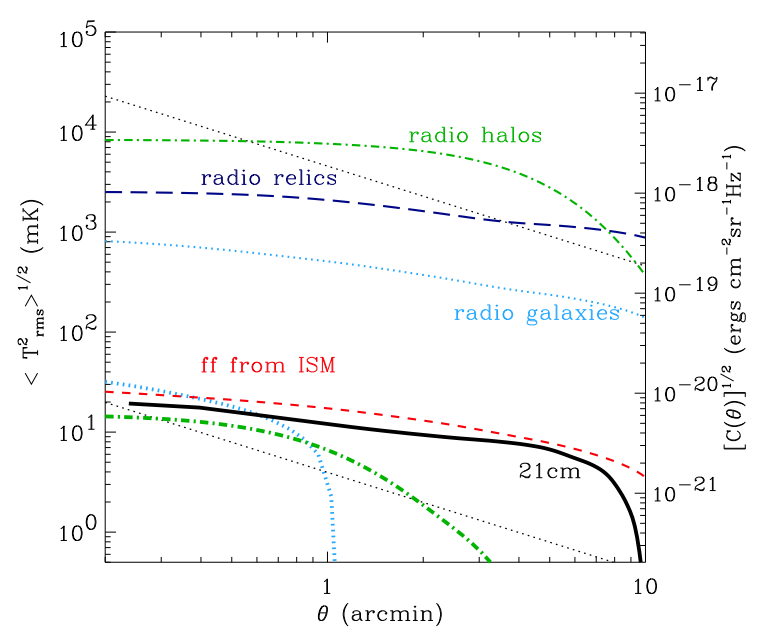

Figure 17: Evolution of the r.m.s. of the brightness temperature of the $21 \mathrm{~cm}$ emission from the IGM (solid line) and from several extra-galactic foregrounds (dashed lines); from top to bottom: radio halos, radio relics, radio galaxies, free-free emission from HII interstellar medium. The curves are calculated at $115 \mathrm{MHz}$. The lower dotted and dashed-dotted curves indicate respectively the radio galaxies and radio halos contamination after removal of sources with flux larger than 0.1 mJy. See Di Matteo, Ciardi \& Miniati 2004 for details.

\section{References}

[1] Ali S. S., Bharadwaj S., Panday B., 2005, MNRAS, 363, 251

[2] Barkana R., Loeb A., 2005, MNRAS, 363, L36

[3] Bland-Hawthorn J., Maloney P. R., 1999, ApJ, 510, 33

[4] Carilli C. L., Gnedin N. Y., Owen F., 2002, ApJ, 577, 22

[5] Carilli C. L., et al., 2004, New A., 48, 1053

[6] Cen R., 2006, ApJ, 648, 47

[7] Chen X., Miralda-Escudé J., 2004, ApJ, 602, 1

[8] Chen X., Miralda-Escudé J., 2006, astro-ph/0605439

[9] Ciardi B., 2007, astro-ph/0709.1367

[10] Ciardi B. et al., 2006, MNRAS, 366, 689

[11] Ciardi B., Bianchi S., Ferrara A., 2002, MNRAS, 331, 463

[12] Ciardi B. \& Ferrara A., 2005, Space Science Rev., 116, 625

[13] Ciardi B., Ferrara A., Governato F., Jenkins A., 2000, MNRAS, 314, 611

[14] Ciardi B., Ferrara A., White S. D. M., 2003, MNRAS, 344, L7

[15] Ciardi B., Madau P., 2003, ApJ, 596, 1

[16] Ciardi B., Stoehr F., White S. D. M., 2003, MNRAS, 343, 1101

[17] Cooray A., Furlanetto S. R., 2005, MNRAS, 359, L47 
[18] Di Matteo T., Ciardi B., Miniati F., 2004, MNRAS, 355, 1053

[19] Di Matteo T., Perna R., Abel T., Rees M. J., 2002, ApJ, 564, 576

[20] Dove J. B., Shull J. M., Ferrara A., 2000, ApJ, 531, 846

[21] Fan X., et al. 2006, AJ, 132, 117

[22] Field, G. B. 1958, Proc. IRE, 46, 240

[23] Fujita A., Martin C., Mac Low M.-M., Abel T., 2003, ApJ, 599, 50

[24] Furlanetto S.R., Oh S.P., Briggs F.H., 2006, Ph. Rev., 433, 181

[25] Glover S. C. O., Brand, P. W. J. L., 2001, MNRAS, 321, 385

[26] Gnedin N. Y., Shaver P. A., 2004, ApJ, 608, 611

[27] Hurwitz M., Jelinsky P., Dixon W. V., 1997, ApJ, 481, L31

[28] Kuhlen M., Madau P., 2005, MNRAS, 363, 1069

[29] Iliev I. T. et al., 2006, MNRAS, 371, 1057

[30] Leitherer C., Ferguson H. C., Heckman T. M., Lowental J. D., 1995, ApJ, 454, L19

[31] Lewis A., Challinor A., astro-ph/0702600

[32] Liu J., et al., 2007, astro-ph/0703330

[33] Loeb A., Zaldarriaga M., 2004, Phys. Rev. Lett., 92, 211301

[34] Madau P., Meiksin A., Rees M. J., 1997, ApJ, 475, 429

[35] Oh S. P., Mack K. J., 2003, MNRAS, 346, 871

[36] Page L. et al., 2007, ApJS, 170, 335

[37] Pillepich A., Porciani C., Matarrese S., 2007, astro-ph/0611126

[38] Rhook K. J., Haehnelt M. G., 2007, MNRAS, 373, 623

[39] Ricotti M., Gnedin N. Y., Shull J. M., 2002, ApJ, 575, 49

[40] Ricotti M., Shull J. M., 2000, ApJ, 542, 548

[41] Salvaterra R., Ciardi B., Ferrara A. \& Baccigalupi C., 2005, MNRAS, 360, 1063

[42] Santos M. G., Cooray A., Knox L., 2005, ApJ, 625, 575

[43] Shapiro P.R., Giroux M.L., 1987, ApJ, 321, 107

[44] Shaver P., Windhorst R., Madau P., de Bruyn G., 1999, A\&A, 345, 380

[45] Steidel C. C., Pettini M., Adelberger K. L., 2001, ApJ, 546, 665

[46] Valdes M., Ciardi B., Ferrara A., Johnston-Hollitt M., Rottgering H., 2006, MNRAS, 369, 66

[47] Wang X., Tegmark M., Santos M. G., Knox L., 2006, ApJ, 650, 529

[48] Wood K., Loeb A., 2000, ApJ, 545, 86

[49] Wouthuysen S. A., 1952, AJ, 57, 31

[50] Wyithe J. S. B., Loeb A., Barnes D. G., 2005, ApJ, 634, 715

[51] Zaldarriaga M., Furlanetto S. R., Hernquist L., 2004, ApJ, 608, 622 
[52] Zaroubi S., Silk J., 2005, MNRAS, 360, 64

[53] Zaroubi S., Thomas R. M., Sugiyama N., Silk J., 2006, astro-ph/0609151

[54] Yoshida N., Abel T., Hernquist L., Sugiyama N., 2003, ApJ, 592, 645

[55] Yoshida N., Omukai K., Hernquist L., 2007, astro-ph/0706.3597 